\title{
Adsorption of Benzene on 4H-SiC (0001) Surface: First Principles Calculations
}

\author{
K. TOKÁR And J.A. MAJEWSKI \\ Institute of Theoretical Physics, Faculty of Physics, University of Warsaw \\ Hoża 69, 00-681 Warsaw, Poland
}

\begin{abstract}
We study adsorption of the benzene molecule on the Si terminated (0001) surface of $4 \mathrm{H}-\mathrm{SiC}$ by performing first principles calculations in the framework of density functional theory. We find out that chemical reaction leading to the chemisorption of benzene on the surface has endothermic character. The adsorbed benzene molecule is bounded to two surface Si atoms and it does not lose its integrity, however, it undergoes strong deformations and causes distortion of the substrate. We analyze also changes in the electronic structure caused by benzene adsorption.
\end{abstract}

PACS: 68.43.-h, 73.20.-r, 77.84.Jd, 31.15.A-

\section{Introduction}

In recent years, graphene has become promising material for novel electronic and optoelectronics devices of the future $[1,2]$. However, the future commercialization of graphene based devices heavily depends on the possibility to grow epitaxial graphene layers of sufficiently good quality [3]. Presently, the growth technique of choice seems to be chemical vapor deposition (CVD) employing various hydrocarbons (such as methane, propane, or/and benzene) as precursors deposited on metallic or silicon carbide surfaces $[4,5]$. The hydrocarbons, in a series of chemical reactions catalyzed by the presence of surface, usually lose their hydrogens and provide carbon atoms that build graphene layers. These processes are subject of intensive experimental and theoretical investigations $[1,6-9]$. In the present paper we report results of our theoretical studies of the adsorption of benzene molecule $\left(\mathrm{C}_{6} \mathrm{H}_{6}\right)$ on silicon terminated (0001) unreconstructed $4 \mathrm{H}-\mathrm{SiC}$ surface. This can be considered as modeling of the very early stages of the graphene's growth by CVD technique using benzene as precursor.

There is a potential advantage of using the benzene as a precursor in the graphene growth over other hydrocarbons. Benzene's core has hexagonal symmetry and its $\mathrm{C}-\mathrm{C}$ bond lengths are close to these in graphene. There exists rather extensive literature on the benzene deposition on metallic surfaces $[10,11]$, but also deposition of benzene on semiconductor (111) silicon surface [12] has been reported. We are also aware of experimental studies dealing with expansion of benzene to giant graphenes [13] and investigating deposition of benzene on SiC surface [14] to grow graphene layers.

The microscopic mechanisms of interactions of the benzene molecules with the metallic and semiconductor surfaces are only weakly known. Very recently, the nu- cleation of benzene on the $\mathrm{Ni}$ surface has been studied theoretically within tight-binding approximation to the density functional theory [15]. In this paper, we employ first-principles calculations to study adsorption of the benzene molecule on the $4 \mathrm{H}-\mathrm{SiC}$ (0001) surface. We calculate adsorption energy, equilibrium geometry, and the changes in the electronic structure caused by the benzene adsorption.

\section{Calculation details}

The calculations of the adsorbed benzene molecule on the (0001) SiC surface have been performed employing slab approach within supercell geometry. In such a slab structure, the active ideal Si surface is unsaturated (i.e., with dangling bonds), whereas the bottom $\mathrm{C}(000-1)$ surface of the slab has been saturated with hydrogen monolayer, just to simulate the situation in the bulk. Since we use numerical procedure that requires periodicity of the system, the slab has been placed in a supercell with $3 \times 3 \times 2$ periodicity and approximately $40 \AA$ of vacuum over the Si plane. This guarantees that the spurious interaction between slab's surfaces is negligible.

The benzene molecule has been placed on the (0001) Si surface and the calculations of the total energy and equilibrium geometry have been performed within the Kohn-Sham realization of the density functional theory as implemented in the localized orbital based package SIESTA [16]. The exchange-correlation functional has been taken in the generalized gradient approximation form of the Perdew-Burke-Ernzerhof (PBE) [17], and the Troullier-Martins pseudopotentials [18] have been chosen to account for electron-ion interaction. We have used the kinetic energy cut off for the wave function of $120 \mathrm{Ry}$ and the Brillouin zone (BZ) integration has been performed 
on $8 \times 8 \times 1$ Monkhorst-Pack [19] sampling mesh, resulting with 32 irreducible special $\boldsymbol{k}$-points. Structural relaxations of atomic positions has been stopped, when the maximal atomic force on all slab atoms has dropped below $0.04 \mathrm{eV} / \AA$.

\section{Results and discussion}

Here, we investigate whether the benzene molecule can be chemisorbed to the $\mathrm{Si}$ terminated $4 \mathrm{H}-\mathrm{SiC}$ surface. We start the computation of the equilibrium geometry of the adsorbed benzene molecule by positioning the free benzene molecule over the $4 \mathrm{H}-\mathrm{SiC}(0001)$ in a random position over Si surface (with $3 \times 3$ surface unit cell). This surface has unsaturated Si dangling bonds that constitute the reactive units of the structure [20]. Then the full relaxation of the atoms in the slab is performed and the equilibrium geometry is obtained as depicted in Fig. 1. We are now in the position to calculate adsorption energy of benzene molecule on the Si terminated $4 \mathrm{H}-\mathrm{SiC}$ surface, which we calculate according to the formula

$$
E_{\mathrm{ads}}=E_{\mathrm{mol}+\mathrm{slab}}-\left(E_{\mathrm{mol}}+E_{\mathrm{slab}}\right),
$$

where $E_{\text {mol+slab }}$ denotes calculated electronic total ground state energy of the optimized configuration of benzene molecule adsorbed on the slab surface, $E_{\text {slab }}$ is the total energy of the slab with clean surface, and $E_{\mathrm{mol}}$ is the energy of free benzene molecule. It turns out that the adsorption energy is equal to $E_{\text {ads }}=-5.2 \mathrm{eV}$ per molecule in the $3 \times 3$ surface unit cell. Thus, benzene experiences an exothermic reaction with the Si surface that leads to chemisorption of benzene. In the calculated stable configuration, the benzene molecule is bonded to two surface $\mathrm{Si}$ atoms, keeping simultaneously all hydrogen atoms bonded to the benzene's carbon atoms. The length of $\mathrm{C}-\mathrm{Si}$ bonds between the benzene core carbons and surface Si layer atoms is equal to $1.92 \AA$, which is very similar value to the typical C-Si bond length (1.92$1.94 \AA$ ) inside the $4 \mathrm{H}-\mathrm{SiC}$ bulk substrate. The benzene core has significantly distorted geometry and $\mathrm{C}-\mathrm{C}$ bonds in core hexagonal ring are variably stretched as shown in Table and in Fig. 1. The $\mathrm{C}-\mathrm{C}$ bond lengths of adsorbed benzene molecule are somewhat larger than in free benzene molecule or in graphene $(1.395 \AA$ and $1.42 \AA$, respectively). The calculated typical $\mathrm{C}-\mathrm{H}$ bond lengths are of the order of $1.10 \AA$. As one can see from these structural characteristics, the adsorbed benzene core has completely broken its original hexagonal symmetry and planar shape. Also the Si and C atoms on the slab surface undergo considerable relaxation along the $c$-axis.

We have also investigated how the chemisorption of benzene molecule changes the electronic structure of the system. For this comparison, we plot the density of states (DOS) of the slab representing pure $\mathrm{SiC}$ surface and slab with the chemisorbed benzene molecule in Fig. 2.

As one can easily see in this figure, the clean Si terminated (0001) surface of $4 \mathrm{H}-\mathrm{SiC}$ is metallic, exhibiting non-zero density of states around the Fermi energy with the width of roughly $3 \mathrm{eV}$. Adsorption of $\mathrm{C}_{6} \mathrm{H}_{6}$ causes

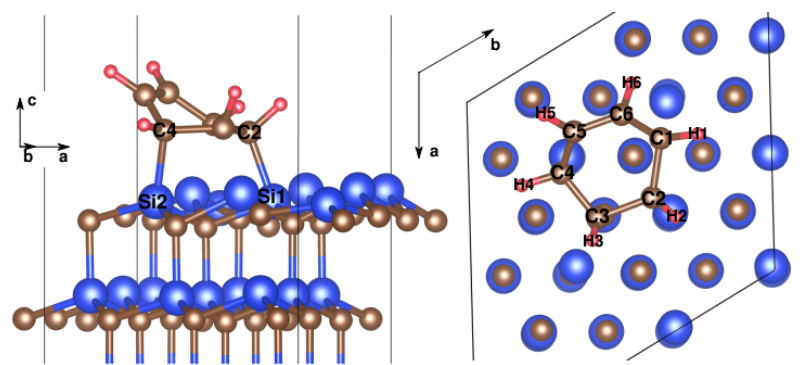

Fig. 1. Side and top view of the chemisorbed benzene molecule on $\mathrm{Si}$ terminated $4 \mathrm{H}-\mathrm{SiC}$ (0001) surface. Large blue circles denote $\mathrm{Si}$ atoms while carbon atoms are shown as medium sized brown circles and hydrogen atoms as small red circles. The black line depicts $3 \times 3$ surface unit cell.

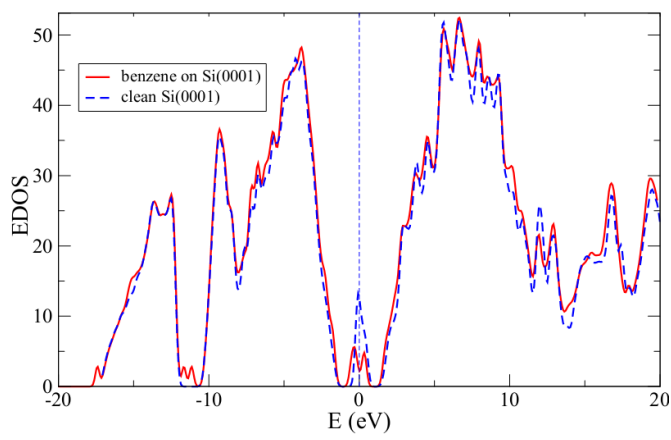

Fig. 2. Density of states for $4 \mathrm{H}-\mathrm{SiC}$ slab with clean (0001) surface (blue dashed line) and of slab with chemisorbed benzene molecule (continuous red line). The bands are shifted in energy, so that the Fermi levels coincide and are placed at zero of energy.

hybridization of surface orbitals with the local orbitals of the adsorbent, which further causes the splitting of central surface band of clean surface into two peaks with approximately the same width.

In the case of isolated benzene, the DOS contains contribution from twelve $\sigma$ and three $\pi$ bonds [20]. It is interesting to compare the DOS of free benzene molecule with the one of deformed $\mathrm{C}_{6} \mathrm{H}_{6}$ molecule adsorbed on the surface. To do this, we compute the projected density of

TABLE

Calculated atomic bond lengths in distorted benzene molecule chemisorbed on the clean $4 \mathrm{H}-\mathrm{SiC}(00001)$ surface, and $\mathrm{Si}-\mathrm{C}$ bond lengths of between benzene's carbons and surface Si atoms (Si1-C2 and Si2-C4). For comparison, the bond lengths in free benzene and graphene are enlisted.

\begin{tabular}{c|c|c|c|c|c}
\hline \hline $\mathrm{C}-\mathrm{C}$ & {$[\AA]$} & $\mathrm{C}-\mathrm{H}$ & {$[\AA]$} & Various bonds & {$[\AA]$} \\
\hline $\mathrm{C} 1-\mathrm{C} 2$ & 1.619 & $\mathrm{C} 1-\mathrm{H} 1$ & 1.116 & $\mathrm{Si} 1-\mathrm{C} 2$ & 1.911 \\
$\mathrm{C} 2-\mathrm{C} 3$ & 1.582 & $\mathrm{C} 2-\mathrm{H} 2$ & 1.110 & Si2-C4 & 1.924 \\
$\mathrm{C} 3-\mathrm{C} 4$ & 1.552 & $\mathrm{C} 3-\mathrm{H} 3$ & 1.107 & $\mathrm{C}-\mathrm{C}$ free benz. & 1.395 \\
$\mathrm{C} 4-\mathrm{C} 5$ & 1.500 & $\mathrm{C} 4-\mathrm{H} 4$ & 1.121 & $\mathrm{C}-\mathrm{C}$ graphene & 1.420 \\
$\mathrm{C} 5-\mathrm{C} 6$ & 1.362 & $\mathrm{C} 4-\mathrm{H} 5$ & 1.104 & $\mathrm{C}-\mathrm{H}$ free benz. & 1.084 \\
$\mathrm{C} 6-\mathrm{C} 1$ & 1.493 & $\mathrm{C} 6-\mathrm{H} 6$ & 1.102 & &
\end{tabular}




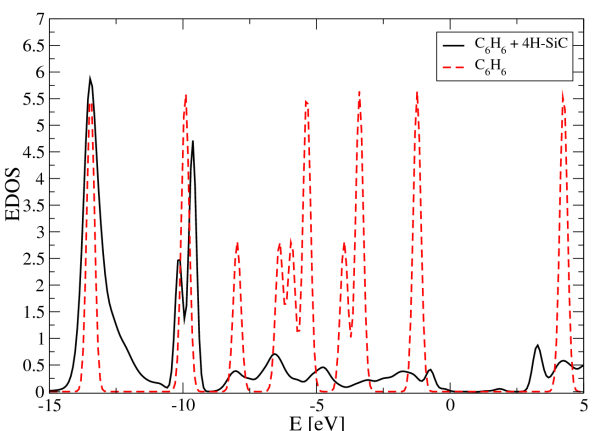

Fig. 3. Comparison of the local density of states for free benzene molecule (blue dashed line) and adsorbed $\mathrm{C}_{6} \mathrm{H}_{6}$ deformed molecule on the Si terminated $4 \mathrm{H}-\mathrm{SiC}$ (0001) surface (solid red line). The intensity of projected adsorbate states in benzene $+4 \mathrm{H}$-slab system is rescaled. Bands of free benzene molecule are shifted in energy to match the lowest state of the deformed $\mathrm{C}_{6} \mathrm{H}_{6}$ adsorbate system.

states using the local orbitals of the adsorbent. This comparison is depicted in Fig. 3. As one can see, the local electronic structure of adsorbed $\mathrm{C}_{6} \mathrm{H}_{6}$ is strongly affected by bonding to the $4 \mathrm{H}-\mathrm{SiC} \mathrm{Si}$ surface. Originally isolated molecular bands of free benzene molecule have been transformed to a continuous band in the $(-8.5-0 \mathrm{eV})$ interval as shown in Fig. 3. There are positioned about ten pronounced peaks induced by the adsorbent between -14 and $0 \mathrm{eV}$. At energy of $-13.4 \mathrm{eV}$, the $\mathrm{C}_{6} \mathrm{H}_{6}$ molecular peak is rather smeared due to adsorbent hybridization, while at $-10 \mathrm{eV}$ the matching adsorbent band peak is doubly degenerated. The rest of adsorbent-induced peaks lies in the continuous part of wide band between -8.5 and $-1 \mathrm{eV}$. From the adsorbent local electronic energy spectra, we deduce that the $\sigma \mathrm{C}-\mathrm{H}$ bonds are slightly influenced by adsorption of benzene on the Si surface, as it was previously observed in the case of benzene symmetrically bound to SiC surface through Si dimer [20].

\section{Conclusions}

We have investigated energetics of the adsorption of the benzene molecule on the $4 \mathrm{H}-\mathrm{SiC}$ (0001) surface by DFT-GGA calculations. We find out that the adsorption energy of benzene molecule to the surface is negative and that the adsorbed benzene molecule does not lose its integrity. The chemical reaction leading to deposition of benzene molecule exhibits exothermic behavior. The absorbed benzene molecule is bonded to two $\mathrm{Si}$ upper layer atoms of $4 \mathrm{H}-\mathrm{SiC}(0001)$ surface, it keeps all six hydrogen atoms, however, its geometry is strongly distorted. Lengths of adsorbent-surface bonds are similar in value with the $\mathrm{Si}-\mathrm{C}$ lengths in the $4 \mathrm{H}-\mathrm{SiC}$ substrate, whereas the $\mathrm{C}-\mathrm{C}$ bonds in the adsorbed benzene core are generally larger than in free molecule or in the graphene. Local electronic structure of adsorbed $\mathrm{C}_{6} \mathrm{H}_{6}$ is significantly affected by bonding with $4 \mathrm{H}-\mathrm{SiC}$ surface.

\section{Acknowledgments}

This research was supported by Polish Ministry of Science and Higher Education "SiCMAT" project POIG.01.03.0114155/0900 and calculations were performed at ICM, University of Warsaw, project no. G47-4.

\section{References}

[1] P.N. First, W.A. de Heer, T. Seyller, C. Berger, J.A. Stroscio, J.-S. Moon, MRS Bull. 35, 296 (2010).

[2] P. Pasanen, M. Voutilainen, M. Helle, X. Song, P.J. Hakonen, Phys. Scr. 2012, 014025 (2012).

[3] Y.H. Wu, T. Yu, Z.X. Shen, J. Appl. Phys. 108, 071301 (2010).

[4] W. Strupinski, K. Grodecki, A. Wysmolek, R. Stepniewski, T. Szkopek, P.E. Gaskell, A. Grüneis, D. Haberer, R. Bozek, J. Krupka, J.M. Baranowski, Nano Lett. 11, 1786 (2011).

[5] Z. Li, P. Wu, C. Wang, X. Fan, W. Zhang, X. Zhai, C. Zeng, Z. Li, J. Yang, J. Hou, ACS Nano 5, 3385 (2011).

[6] A. Catellani, A. Calzolari, J. Phys. Chem. C 116, 886 (2012).

[7] R. Di Felice, C.A. Pignedoli, C.M. Bertoni, A. Catellani, P.L. Silvestrelli, C. Sbraccia, F. Ancilotto, M. Palummo, O. Pulci, Surf. Sci. 532-535, 982 (2003).

[8] H. Kageshima, H. Hibino, M. Nagase, H. Yamaguchi, Appl. Phys. Expr. 2, 065502 (2009).

[9] A.N. Hattori, T. Okamoto, S. Sadakuni, J. Murata, K. Arima, Y. Sano, K. Hattori, H. Daimon, K. Endo, K. Yamauchi, Surf. Sci. 605, 597 (2011).

[10] G.-P. Dai, P.H. Cooke, S. Deng, Chem. Phys. Lett. 531, 191 (2012).

[11] Z. Li, P. Wu, Ch. Wang, X. Fan, W. Zhang, X. Zhai, Ch. Zeng, Z. Li, J. Yang, J.G. Hou, arXiv: 1101.5664 v2.

[12] H. Tomimoto, T. Takehara, K. Fukawa, R. Sumii, T. Sekitani, K. Tanaka, Surf. Sci. 526, 341 (2003).

[13] S. Müller, K. Müllen, Philos. Trans. R. Soc. A $\mathbf{3 6 5}$, 1453 (2007).

[14] A.J. Oyer, J.-M.Y. Carrillo, Ch.C. Hire, H.C. Schniepp, A.D. Asandei, A.V. Dobrynin, D.H. Adamson, J. Am. Chem. Soc. 134, 5018 (2012).

[15] Ch. Zhou, J. Hu, Y. Tian, Q.-Y. Zhao, L. Miu, J.-J. Jiang, J. At. Mol. Sci. 3, 270 (2012).

[16] J.M. Soler, E. Artacho, J.D. Gale, A. García, J. Junquera, P. Ordejón, D.S. Sánchez-Portal, J. Phys., Condens. Matter 14, 2745 (2002).

[17] J.P. Perdew, K. Burke, M. Enzerhof, Phys. Rev. Lett. 77, 3865 (1996).

[18] N. Troullier, J.L. Martins, Phys. Rev. B 43, 1993 (1991).

[19] H.J. Monkhorst, J.D. Pack, Phys. Rev. B 13, 5188 (1976).

[20] J. Wieferink, P. Krüger, J. Pollmann, Phys. Rev. B 78, 165315 (2008). 